\title{
Dielectric property of lead phthalocyanine
}

\author{
K D PATEL and A T OZA* \\ Department of Physics, Sardar Patel University, Vallabh Vidyanagar 388 120, India \\ MS received 29 April 1995; revised 26 June 1995
}

\begin{abstract}
Dielectric constant and dissipation factor of lead phthalocyanine have been experimentally studied here as functions of frequency and temperature. Dielectric constant increases by one order of magnitude with temperature showing pyroelectric behaviour. It is explained that molecular distortion leads to increased dielectric constant.
\end{abstract}

Keywords. Dielectric constant; dissipation factor; molecular distortion; exciton mechanism.

\section{Introduction}

Lead phthalocyanine ( $\mathrm{PbPc}$ ) (tetraphthalonitrile) has lead chains along one crystallographic direction (Ukie 1973). It is found to be semiconducting(Ukie 1975; Frauenheim et al 1984).

Electrical resistivity of lead phthalocyanine in the form of thin films remains constant down to $4.2 \mathrm{~K}$ (Ukie 1975). Here we report dielectric property of this quasi one dimensional conductor.

\section{Experimental}

Lead tetraphthalonitrile (phthalocyanine) was prepared by refluxing lead monoxide with phthalonitrile from solution in nitrobezene (figure 1). The material was prepared from $\mathrm{PbO}$ and phthalonitrile powders both of which were amorphous. Thus $\mathrm{PbPc}$ was prepared in amorphous form. After that it was ground in a mortar to remove grains due to moisture and it was compressed in a high pressure die. So PbPc pellets were amorphous and not polycrystalline. A sample holder as shown (figure 2) was used for measurement of capacitance. The dielectric constant was evaluated from $C=C_{0} \varepsilon$ where $C_{0}=\varepsilon_{0} A / d . \varepsilon_{0}$ is dielectric constant of air, $A$ the area of pellet of $\mathrm{PbPc}$ and $d$ the thickness of the pellet $\left\{\Sigma(100 \mathrm{~Hz})=93\right.$ at $\left.40^{\circ} \mathrm{C}\right\}$. Capacitance and dissipation factors (dielectric loss) were measured using multifrequency LCR meter of Hewlett Packard Co.

\section{Results and discussion}

Both capacitance and dissipation factors are plotted against frequency and temperature (figures 3-6). The capacitance was found to increase in the range $40^{\circ} \mathrm{C}-300^{\circ} \mathrm{C}$ at $1 \mathrm{kHz}$. This temperature dependence reveals pyroelectric behaviour. When dielectric constant varies with temperature and remnant polarization takes place, then it is called a pyroelectric material (Tareev 1974).

There can be several contributions to dielectric constant viz. (i) ionic polarizability, (ii) interstitial polarization, (iii) electronic polarizability of atoms, (iv) lattice polarization, 


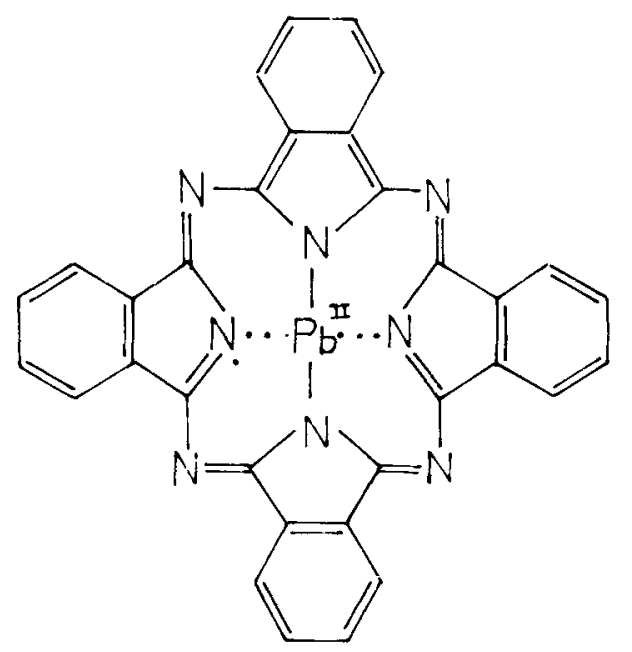

Figure 1. Molecular structure of lead phthalocyanine.

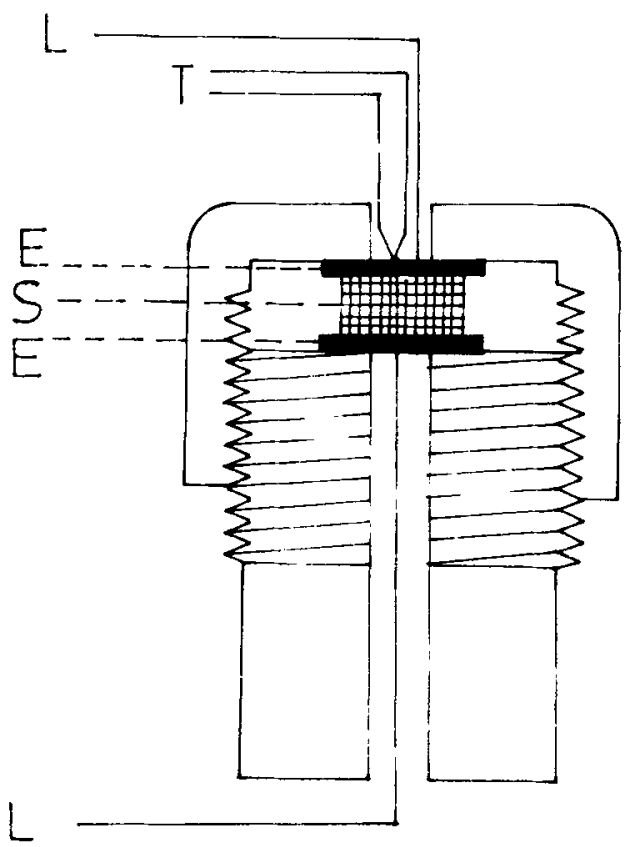

Figure 2. Sample holder for capacitance measurement.

(v) molecular polarizability and (vi) ferroelectric transition. We discard the possibility of ion core polarizability because in that case dielectric constant should not change with temperature.

Interstitial polarization is also discarded because of homogeneous and amorphous pellets which are a continuous medium. The contribution from electronic polarizability is negligible because in that case variation of dielectric constant with frequency 


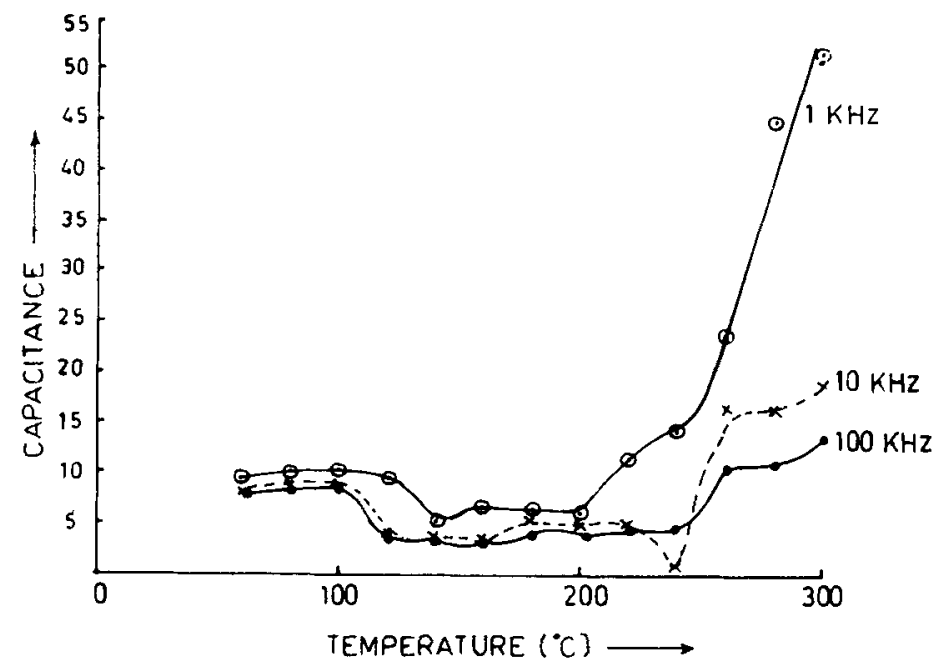

Figure 3. Temperature dependence of dielectric constant (capacitance is in pf).

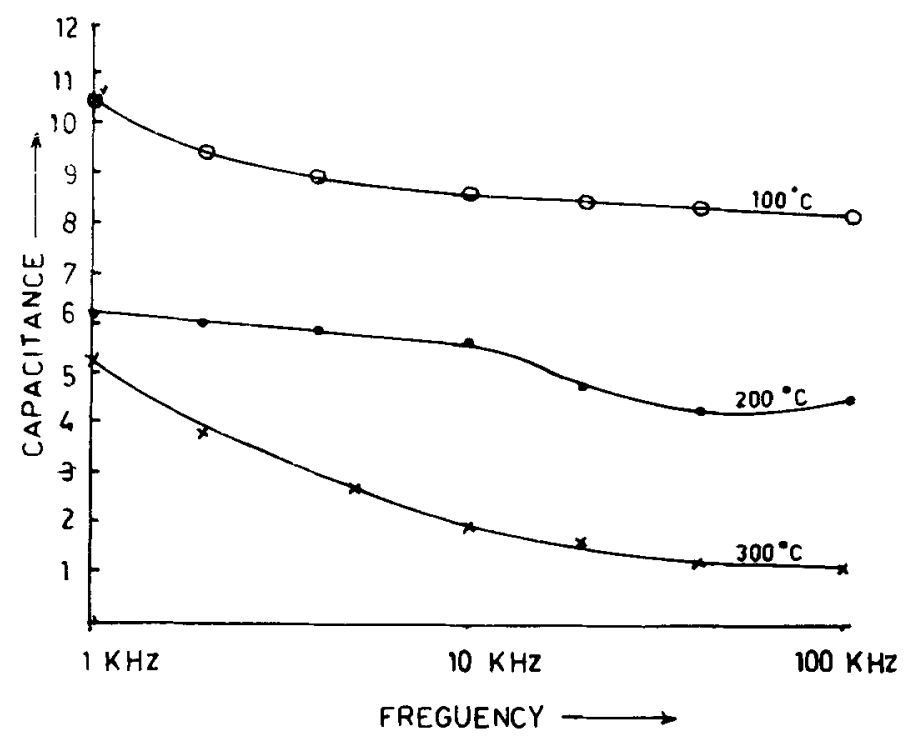

Figure 4. Frequency dependence of dielectric constant (capacitance is in pf).

should be very large. But it is not large (figure 4). Lattice polarizability is also not possible because of neutral molecules of $\mathrm{PbPc}$ in each unit cell. Also ferroelectric transition is discarded because change is only one order of magnitude and because of absence of two types of ions. Dielectric constant does not arise from localization of electrons.

There is no strong electron-phonon interaction along lead chains because of large band gap (Frauenheim et al 1984). Phonon generation at high temperature should increase dielectric constant due to phonon drag. Therefore, phonon drag does not localize charge carriers. Dielectric constant is not found to increase with frequency and 


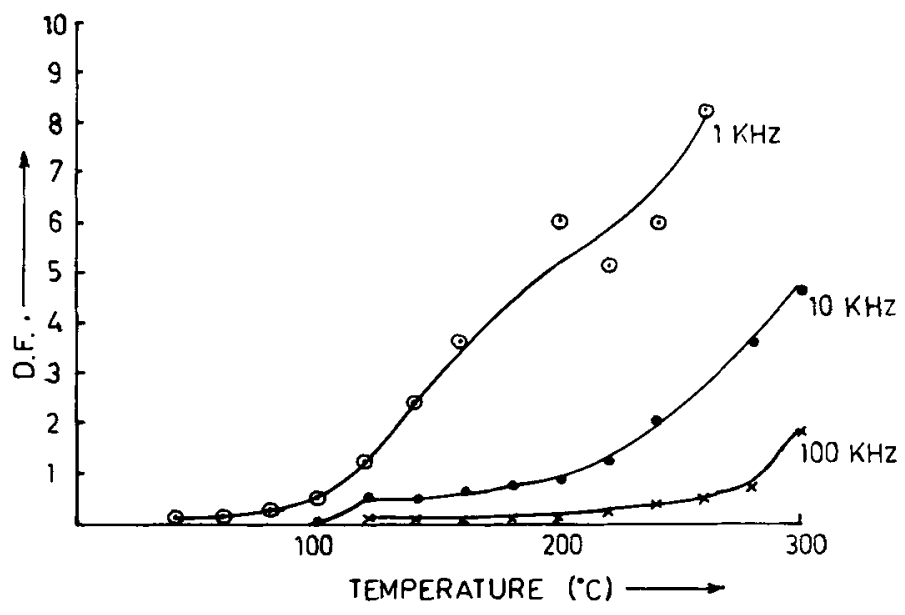

Figure 5. Temperature dependence of dissipation factor.

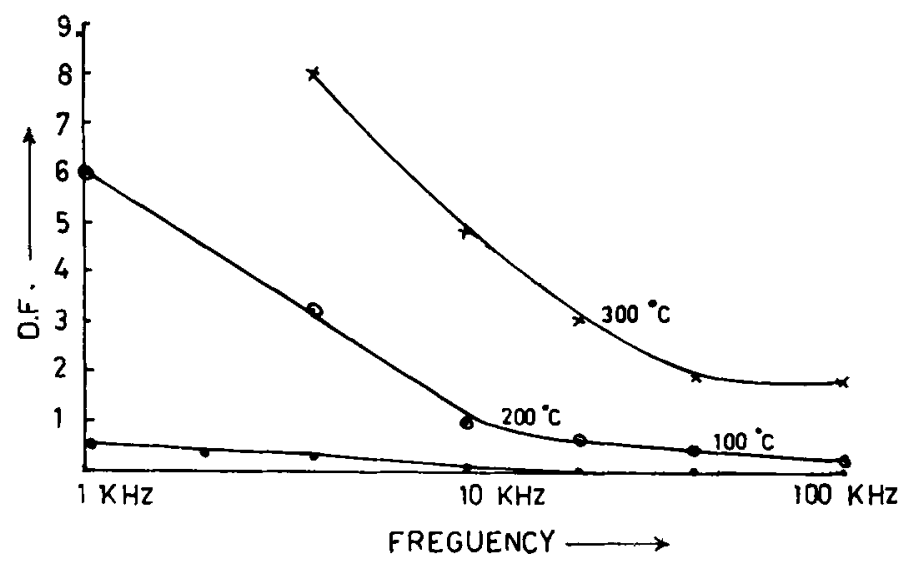

Figure 6. Frequency dependence of dissipation factor.

this discards the possibility of any kind of dispersion. Also there are no localized states along lead chains in which case a trap-limited localization governs.

In an inductor or capacitor the DF is the cotangent of the phase angle $\alpha$ or the tangent of the loss angle $\varepsilon$. In low loss components, it is almost equal to power factor $\cos \alpha$ and can be given approximately by $\sigma / 2 \pi f E$. The dissipation causes free oscillations to be damped and removes sharpness of cut off in filters. This is the displacement polarization energy. The dissipated energy goes to heating effect.

Dissipation factor varies very similar to dielectric constant with temperature and frequency. It decreases by one order of magnitude with frequency showing considerable dispersion at $300^{\circ} \mathrm{C}$.

One possibility is molecular polarizability. Two electrons in $\mathrm{PbPc}$ molecule show correlated motions and pairing as suggested for such a molecule (Kampas 1970). These electrons are in $5 d_{x^{2}-y^{2}}$ orbital (figures $5 \mathrm{a}$ and b). Molecule of PbPc is distorted in a $5 d_{x^{2}-y^{2}} 6 S^{1}$ or $6 P^{1}$ transition because of phonon absorption at higher temperature. 
(a)
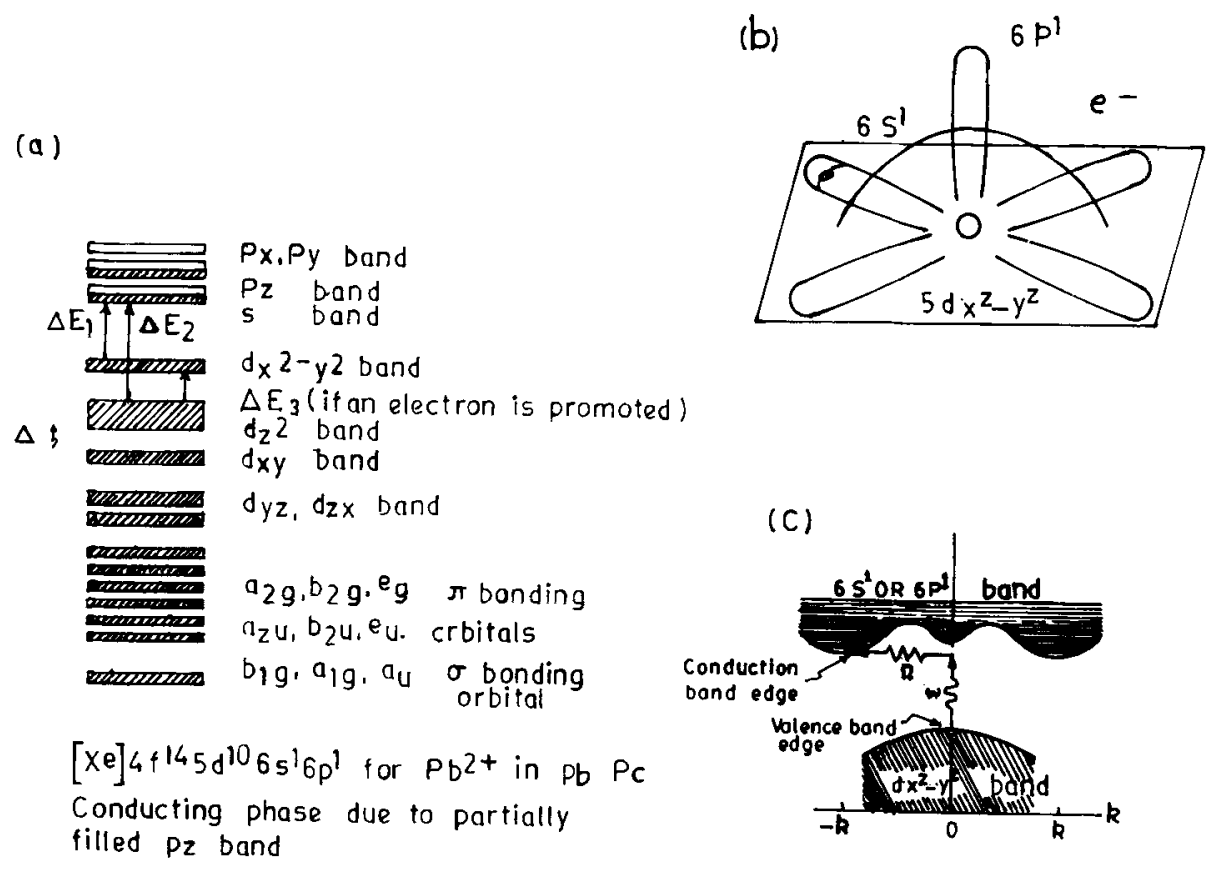

Figure 7. (a) Band structure of $\mathrm{PbPc}$, (b) $5 d_{x^{2}-y^{2}} \rightarrow 6 S^{1}$ or $6 S^{1}$ transition and (c) indirect band gap.

This is an intermolecular transition. Orthogonality of the orbitals is related with relative shift of conduction and valence band along wave-vector axis. This leads to an indirect transition involving a phonon absorption (figure 5c). Molecule is left in polarized or deformed state involving quinonoid resonance. Each distorted molecule has large dipole moment in this excited state and electrons are decoupled with optical phonons of intramolecular vibrations. Conducting lead chains are embedded in a medium of high dielectric constant as required in excitonic mechanism of high temperature superconductivity (Davis et al 1976).

Phonon energy can be calculated from uncertainty relation as

$$
E_{P}=(\Delta P)^{2} / 2 m
$$

where $\Delta P \cong \hbar / \Delta x$ and $x$ is half of the diagonal size of molecule for $d_{x^{2}-y^{2}} \rightarrow 6 P$ transition (figure 7). This comes out to be $0.03 \mathrm{eV}$ which is approximately the same energy as thermal energy supplied between $40^{\circ} \mathrm{C}$ and $300^{\circ} \mathrm{C}$.

There is hybridization of $d_{x^{2}-y^{2}}$ orbital of $\mathrm{Pb}^{2+}$ ion with orbital of phthalocyanine ligand. This is why molecular size is involved in the above calculation. Actually a $\pi$-electron of the ligand transits to $6 S$ or $6 P$ orbital of $\mathrm{Pb}^{2+}$ ions leaving the molecule in distorted state. Molecule stops resonating freely. Electron absorbs phonon with which it has strong coupling. Electron carries away momentum of a molecule in the sense that momentum associated with resonances and vibrations is lost. The distorted molecule is left in a polarized state and has large dipole moment. This increases the overall dielectric constant. Charge carrier migrates to neighbouring mole by carrying away momentum associated with vibrations. 


\section{Conclusion}

There is molecular distortion at high temperature which increases dielectric constant of lead phthalocyanine.

\section{Acknowledgement}

The authors are thankful to Prof. R Srivastava, Department of Physics, SP University, for kind cooperation.

\section{References}

Davis D, Gutfreund H and Little W A 1976 Phys. Rev. B13 4766

Frauenheim Th, Homann C and nu Mueller M 1984 Phys. Status Solidi 86735

Kampas F J 1970 Polym. Symp., J. Polym. Sci. C29 81

Tareev B 1974 Physics of dielectric materials (Moscow: Mir publishers)

Ukie K 1973 Acta Crystallogr. B29 2990

Ukie K 1975 Phys. Lett. A55 111 